\title{
Computer Aided Analysis on Higher-dimensional Nonlocal Reaction Diffusion Population Dynamic Model
}

\author{
Linan Sun ${ }^{1, a}$, Shan $\mathrm{Li}^{1, \mathrm{~b}}$ \\ ${ }^{1}$ Mathematics Department, Heihe University, Heihe 164300, PR China

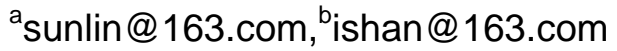

Keywords: Nonlocal influence, Logistic equation, Reaction-diffusion dynamic system, Finite difference method, MATLAB

\begin{abstract}
In this paper, we consider a reaction-diffusion dynamic model which is of the classical Fisher type, but incorporates nonlocal influence into competitive term. We present two finite-difference algorithms for studying the dynamics of nonlocal reaction-diffusion logistic equation. We also present the results of numerical experiments in two space dimensions and illustrate the simplicity of the numerical methods with programs in MATLAB.
\end{abstract}

\section{Introduction}

Population dynamics is a branch of ecology and it covers a wide spectrum of fields. Many examples show the significance of population ecology. The formation and the evolution of natural patterns provides many examples, see for example [1, 2]. The Fisher equation [3, 4], which combines the diffusive motion of individuals with nonlinearities arising from their growth and competition process, has served as a unifying entity in various mathematical investigations in ecology and biology. The equation can be written as follows: [5]

$$
\frac{\partial u(x, t)}{\partial t}=D \frac{\partial^{2} u(x, t)}{\partial x^{2}}+a u(x, t)-b u^{2}(x, t)
$$

Where $D$ is the coefficient of the diffusion process, $a$ is the rate of population growth, and $b$ controls the limiting process. The equation describes the temporal and spatial evolution of the density of individuals such as bacteria or rodents, and it not only focuses on some processes such as reproduction, competition for resources and diffusion, but also considers other complex processes such as mutation and predator-prey interaction.

The Fisher equation only considers local effects in the competition terms. However, in the reality, individuals sometimes compete for resource not only in their immediate neighborhood but also in more board domain. For that reason, many people begin to generalize the equation by incorporating nonlocal effects in the competition terms. The generalized equation on a spatial domain with arbitrary dimensions has the following form: [6]

$$
\frac{\partial u(x, t)}{\partial t}=D \frac{\partial^{2} u(x, t)}{\partial x^{2}}+a u(x, t)-b u(x, t) \int_{\Omega} u(y, t) f_{\sigma}(x, y) d y
$$

where $f_{\sigma}(x, y)$ is a positive distribution function which we call the influence function, characterized by a range $\sigma$ and normalized in the domain $\Omega$ under investigation, $\Omega$ is the domain for the nonlocal interaction. We find that Eq.2 features competitive interactions linking density $u(x, t)$ with density $u(y, t)$ through an influence function $f_{\sigma}(x, y)$ of range $\sigma$. The simplest influence functions are a constant, a Gaussian and a square distribution.

Fuentes et.al. [6,7] have shown that such a generalization leads to interesting patterns in the steady state density. The Fisher equation and its modified version have been applied to bacterial dynamics in Petri dishes and to epidemics particularly in the West Nile virus. Bacterial colonies have been studied [1] experimentally as well as theoretically [8, 9].

In [10], the existence and the stability of the equilibrium solutions of the traditional diffusive logistic equation are studied with analytic and numerical methods. In [11], the properties of steady 
state solutions of nonlocal logistic equation with some constrain on influence function were studied in one-dimension, but not in high-dimension.

Here, we mainly consider the nonlocal logistic equation with square influence function in high-dimensional space $\Omega$, and a zero boundary condition is imposed:

$$
\left\{\begin{array}{lc}
u_{t}=D \Delta u+a u-b u \int_{\Omega_{1}} u(y, t) f_{\sigma}(x, y) d y, & x \in \Omega, t>0, \\
\left.u\right|_{\partial \Omega}=0, & t>0, \\
u(x, 0)=g(x) & x \in \Omega,
\end{array}\right.
$$

where $f_{\sigma}(x, y)=\{\begin{array}{ll}1, & y \in \bar{\Omega}_{1}, x \in \bar{\Omega}, \\ 0, & \text { otherwise, }\end{array}$ and $\bar{\Omega}_{1}=\underbrace{\left[x_{1}-k L, x_{1}+k L\right] \times \cdots \times\left[x_{n}-k L, x_{n}+k L\right]}_{n}, x \in \Omega$,

$k \in(0,1], \bar{\Omega}=[-L, L] \times \cdots \times[-L, L]$.

For the sake of reducing the dependence of parameters, we should do nondimesionalization to Eq.3, after that we obtain a new equation:

$\left\{\begin{array}{lc}u_{t}=\Delta u+\lambda u-\lambda u \int_{\Omega_{3}} u(y, t) f_{\sigma}(x, y) d y, & x \in \Omega_{2}, t>0, \\ \left.u\right|_{\partial \Omega_{2}}=0, & t>0, \\ u(x, 0)=g_{1}(x) & x \in \Omega_{2},\end{array}\right.$

where $\lambda=\frac{a L^{2}}{D}, g_{1}(x)=g(x L) \cdot \frac{b L}{a}$, and $\bar{\Omega}_{3}=\underbrace{\left[x_{1}-k, x_{1}+k\right] \times \cdots \times\left[x_{n}-k, x_{n}+k\right]}_{n}, x \in \Omega_{2},$.

$x=\left(x_{1}, \cdots, x_{n}\right), k \in(0,1], \bar{\Omega}_{2}=[-1,1] \times \cdots \times[-1,1]$.

In the following, for the sake of generalizing the qualitative study to high-dimension space, we will present some algorithms, and we will show some numerical simulations by MATLAB software to promote theory results.

\section{Algorithms}

We will write the algorithms for problem Eq.4 in the following. In this section, we consider the problem Eq.4 in two-dimensional space.

Domain division. We divide the spatial interval $[-1,1] \times[-1,1]$ with grid points $\left(x_{1 i}, x_{2 l}\right)=$ $(i h-1, l h-1), i, l=1, \cdots, n$, where $h$ is the space step size. We also take a subdivision of the time interval $[0, T]$ with time levels $t_{j}=j \Delta t, j=1, \cdots, N$, so the time step is $\Delta t=\frac{T}{N}$.

Difference scheme. In this part, we will construct difference scheme for Eq.4 in two-dimension

$$
u_{t}=\Delta u+\lambda u-\lambda u \int_{\Omega_{3}} u(y, t) d y, x \in[-1,1] \times[-1,1], t>0,
$$

where $\Omega_{3}=\left[x_{1}-k, x_{1}+k\right] \times\left[x_{2}-k, x_{2}+k\right], x=\left(x_{1}, x_{2}\right)$.

We can use the forward finite difference scheme as in one-dimension or P-R difference scheme to discrete Eq.5. First we apply the forward difference scheme. Clearly Eq. 5 can be changed into another equal form:

$$
u_{t}=\Delta u+\lambda u-\lambda u \int_{\Omega_{3}} u(x+z, t) d z, x \in[-1,1] \times[-1,1], t>0,
$$

where $\Omega_{3}^{\prime}=[-k, k] \times[-k, k]$, Eq.6 can be discretelized as follows: 


$$
\left\{\begin{array}{c}
\frac{u_{i l}^{j+1}-u_{i l}^{j}}{\Delta t}=\frac{u_{i+1, l}^{j}-4 u_{i l}^{j}+u_{i-1, l}^{j}+u_{i, l+1}^{j}+u_{i, l-1}^{j}}{(\Delta x)^{2}}+\lambda u_{i l}^{j}-\lambda u_{i l}^{j} \sum_{p=1}^{\left(\frac{k}{\Delta x}-1\right)+i} \frac{u_{p+1, p+1}^{j}+u_{p, p}^{j}+u_{p+1, p}^{j}+u_{p, p+1}^{j}}{4} \cdot \Delta x \cdot \Delta y, \\
i, l=2, \cdots, \frac{k}{\Delta x}+1, j \geq 1, \\
\frac{u_{i l}^{j+1}-u_{i l}^{j}}{\Delta t}=\frac{u_{i+1, l}^{j}-4 u_{i l}^{j}+u_{i-1, l}^{j}+u_{i, l+1}^{j}+u_{i, l-1}^{j}}{(\Delta x)^{2}}+\lambda u_{i l}^{j}-\lambda u_{i l}^{j} \sum_{p=1}^{\left(\frac{k}{\Delta x}-1\right)+i} \frac{u_{p+1, p+1}^{j}+u_{p, p}^{j}+u_{p+1, p}^{j}+u_{p, p+1}^{j}}{4} \cdot \Delta x \cdot \Delta y, \\
i, l=\frac{k}{\Delta x}+2, \cdots, \frac{2-k}{\Delta x}, j \geq 1, \\
\frac{u_{i l}^{j+1}-u_{i l}^{j}}{\Delta t}=\frac{u_{i+1, l}^{j}-4 u_{i l}^{j}+u_{i-1, l}^{j}+u_{i, l+1}^{j}+u_{i, l-1}^{j}}{(\Delta x)^{2}}+\lambda u_{i l}^{j}-\lambda u_{i l}^{j} \sum_{p=1}^{\left(\frac{k}{\Delta x}-1\right)+i} \frac{u_{p+1, p+1}^{j}+u_{p, p}^{j}+u_{p+1, p}^{j}+u_{p, p+1}^{j}}{4} \cdot \Delta x \cdot \Delta y, \\
i, l=\frac{2-k}{\Delta x}+1, \cdots n, j \geq 1,
\end{array}\right.
$$

Dealing with initial condition. $u_{i l}^{1}=g_{1}\left(x_{m}\right), m=i+n l, i, l=2, \cdots, n$.

Dealing with boundary conditions. $u_{1 l}^{j+1}=u_{n+1, l}^{j+1}=u_{i 1}^{j+1}=u_{i, n+1}^{j+1}=0, j=1, \cdots$.

Next we describe the P-R scheme of equation Eq.5 as follows:

$$
\begin{aligned}
& \int \frac{u_{i l}^{j+\frac{1}{2}}-u_{i l}^{j}}{\frac{\Delta t}{2}}=\frac{u_{i+1, l}^{j+\frac{1}{2}}-2 u_{i l}^{j+\frac{1}{2}}+u_{i-1, l}^{j+\frac{1}{2}}+u_{i, l+1}^{j}-2 u_{i l}^{j}+u_{i, l-1}^{j}}{(\Delta x)^{2}}+\lambda u_{i l}^{j}-\lambda u_{i l}^{j} \sum_{p=1}^{\left(\frac{k}{\Delta \alpha}-1\right)+i} \frac{u_{p+1, p+1}^{j}+u_{p, p}^{j}+u_{p+1, p}^{j}+u_{p, p+1}^{j}}{4} \cdot \Delta x \cdot \Delta y, \\
& \frac{u_{i l}^{j+1}-u_{i l}^{j+\frac{1}{2}}}{\frac{\Delta t}{2}}=\frac{u_{i+1, l}^{j+\frac{1}{2}}-2 u_{i l}^{j+\frac{1}{2}}+u_{i-1, l}^{j+\frac{1}{2}}+u_{i, l+1}^{j+1}-2 u_{i l}^{j+1}+u_{i, l-1}^{j+1}}{(\Delta x)^{2}}+\lambda u_{i l}^{j}-\lambda u_{i l}^{j} \sum_{p=1}^{\left(\frac{k}{\Delta x}-1\right)+i} \frac{u_{p+1, p+1}^{j}+u_{p, p}^{j}+u_{p+1, p}^{j}+u_{p, p+1}^{j}}{4} \cdot \Delta x \cdot \Delta y, \\
& i, l=2, \cdots, \frac{k}{\Delta x}+1, j \geq 1 \text {, } \\
& \frac{u_{i l}^{j+1}-u_{i l}^{j}}{\Delta t}=\frac{u_{i+1, l}^{j}-4 u_{i l}^{j}+u_{i-1, l}^{j}+u_{i, l+1}^{j}+u_{i, l-1}^{j}}{(\Delta x)^{2}}+\lambda u_{i l}^{j}-\lambda u_{i l}^{j} \sum_{p=1}^{\left(\frac{k}{\Delta x}-1\right)+i} \frac{u_{p+1, p+1}^{j}+u_{p, p}^{j}+u_{p+1, p}^{j}+u_{p, p+1}^{j}}{4} \cdot \Delta x \cdot \Delta y, \\
& \left\{\frac{u_{i l}^{j+1}-u_{i l}^{j+\frac{1}{2}}}{\frac{\Delta t}{2}}=\frac{u_{i+1, l}^{j+\frac{1}{2}}-2 u_{i l}^{j+\frac{1}{2}}+u_{i-1, l}^{j+\frac{1}{2}}+u_{i,+1}^{j+1}-2 u_{i l}^{j+1}+u_{i, l-1}^{j+1}}{(\Delta x)^{2}}+\lambda u_{i l}^{j}-\lambda u_{i l}^{j} \sum_{p=1}^{\left(\frac{k}{\Delta x}-1\right)+i} \frac{u_{p+1, p+1}^{j}+u_{p, p}^{j}+u_{p+1, p}^{j}+u_{p, p+1}^{j}}{4} \cdot \Delta x \cdot \Delta y,\right. \\
& i, l=\frac{k}{\Delta x}+2, \cdots, \frac{2-k}{\Delta x}, j \geq 1, \\
& \frac{u_{i l}^{j+1}-u_{i l}^{j}}{\Delta t}=\frac{u_{i+1, l}^{j}-4 u_{i l}^{j}+u_{i-1, l}^{j}+u_{i, l+1}^{j}+u_{i, l-1}^{j}}{(\Delta x)^{2}}+\lambda u_{i l}^{j}-\lambda u_{i l}^{j} \sum_{p=1}^{\left(\frac{k}{\Delta x}-1\right)+i} \frac{u_{p+1, p+1}^{j}+u_{p, p}^{j}+u_{p+1, p}^{j}+u_{p, p+1}^{j}}{4} \cdot \Delta x \cdot \Delta y, \\
& \frac{u_{i l}^{j+1}-u_{i l}^{j+\frac{1}{2}}}{\frac{\Delta t}{2}}=\frac{u_{i+1, l}^{j+\frac{1}{2}}-2 u_{i l}^{j+\frac{1}{2}}+u_{i-1, l}^{j+\frac{1}{2}}+u_{i, l+1}^{j+1}-2 u_{i l}^{j+1}+u_{i, l-1}^{j+1}}{(\Delta x)^{2}}+\lambda u_{i l}^{j}-\lambda u_{i l}^{j} \sum_{p=1}^{\left(\frac{k}{\Delta x}-1\right)+i} \frac{u_{p+1, p+1}^{j}+u_{p, p}^{j}+u_{p+1, p}^{j}+u_{p, p+1}^{j}}{4} \cdot \Delta x \cdot \Delta y, \\
& i, l=\frac{2-k}{\Delta x}+1, \cdots n, j \geq 1
\end{aligned}
$$

\section{Experiments}

In this section we use MATLAB program doing some numerical experiments to test some of our qualitative analysis before and motivate some mathematical results we have not obtain. Firstly, for the sake of simplification, we take $\mathrm{k}=0.3$ in Eq. 4 and $n=2$, consider the Dirichlet problem: 


$$
\left\{\begin{array}{lr}
\left.u_{t}=\Delta u+\lambda u-\lambda u \int_{\Omega_{3}} u(y, t)\right) d y, x \in \Omega_{2}, t & >0, \\
\left.u\right|_{\partial \Omega_{2}}=0, & t>0, \\
u(x, 0)=g_{1}(x) & x \in \Omega_{2},
\end{array}\right.
$$

where $\lambda=\frac{a L^{2}}{D}, g_{1}(x)=g(x L) \cdot \frac{b L}{a}$, and $k \in(0,1], \bar{\Omega}_{2}=[-1,1] \times[-1,1]$.

$\bar{\Omega}_{3}=\left[x_{1}-0.3, x_{1}+0.3\right] \times\left[x_{2}-0.3, x_{2}+0.3\right], x \in \Omega_{2}, x=\left(x_{1}, x_{2}\right) x$.

We use the algorithm described firstly in the last section and we take grid size $\Delta x_{1}=0.1$ and $\Delta x_{2}=0.1$. In the program, we assume $\lambda=1$, and $g_{1}(x)=2-\left(x_{1}^{2}+x_{2}^{2}\right)$. When we make the simulation, firstly, we find that the graphic decreasing from the original shape when $t=0$ to the shape when $t=0.1$ (see Fig. 1(a) and (b)). With the time developing, the graphic become a sequence of decreasing arches until $u$ tend to zero for all $x$ in spatial interval when $t$ is large or tend to infinity (see Fig. 1 (c)and (d))

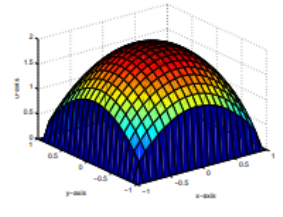

a

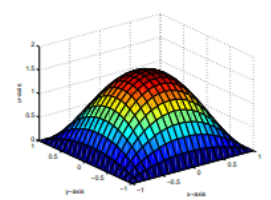

$\mathrm{b}$

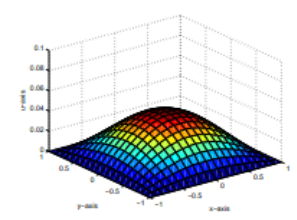

C

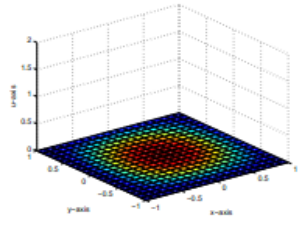

d

Fig. 1 Graph of the solution of Eq.4 when $t=0, t=0.1, t=1, t=10$ in turn and for the four

$$
\text { cases } \lambda=1, u(x, 0)=2-\left(x_{1}^{2}+x_{2}^{2}\right) \text {. }
$$

In above, we take $\lambda=1$ which is smaller than $\pi^{2} / 2$, and the experiment result meets with the theory conclusion in [12]. Next we will consider $\lambda>\pi^{2} / 2$. Let us change the parameter to $\lambda=10$ and try two difference initial data: $(1) u(x, 0)=2-\left(x_{1}^{2}+x_{2}^{2}\right)$ and $(2) u(x, 0)=\cos \frac{\pi x_{1}}{2} \cos \frac{\pi x_{2}}{2}$.

When we make simulation, we can find that in the first case, the solution become the graphic in (Fig. 2 (a)), and in the second case, the solution become the graphic in (Fig. 2 (b)) for a short time. Let us increase the time iterations to $t=10$, we can see that it is almost identical for two cases (Fig. 2 (c) and (d)).

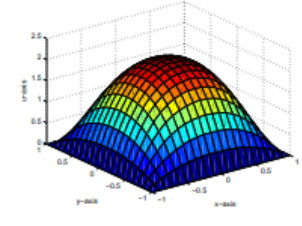

a

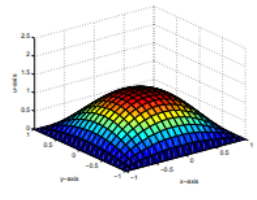

$b$

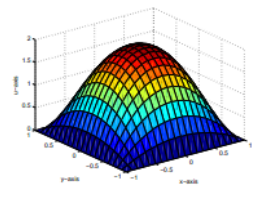

c

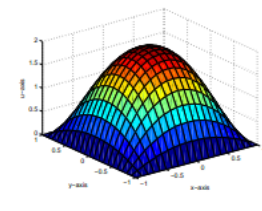

d

Fig. 2 a. and b. Graph of the solution of Eq.4 when $t=0.1, \lambda=10$ with initial data (1) and (2); c. and d. Graph of the solution of Eq.4 when $t=10, \lambda=10$ with initial data (1) and (2).

Hence, from the experiment for $\lambda=10$, we find the solution finally will approach a limit function with maximum value at about $u=1.983$. This limit function doesn't change when $t$ changes, thus it must be a solution of the equation and it satisfies

$$
\left\{\begin{array}{l}
\left.\Delta u+\lambda u-\lambda u \int_{\Omega_{3}} u(y, t)\right) d y=0, x \in \Omega_{2}, \\
\left.u\right|_{\partial_{\mathrm{O}_{2}}}=0
\end{array}\right.
$$

For more large $\lambda$, we will find similar phenomena except the equilibrium solutions have different profiles. When $\lambda=100$, the profile of the equilibrium solution is as (Fig. 3 (a)), and when $\lambda=300$, as (Fig. 3 (b)). 


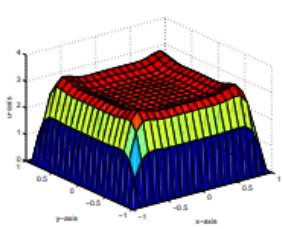

a

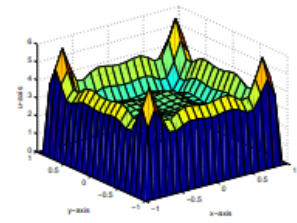

b

Fig. 3 Graph of the solution of Eq. 4 when $t=10, \lambda=100, \lambda=300, u(x, 0)=2-\left(x_{1}^{2}+x_{2}^{2}\right)$.

We choose different parameters $\lambda$ for simulation and write the maximal $\|u\|_{\infty}$ at equilibrium state. So we obtain the bifurcation diagram of the equilibrium solutions of Eq.4 as follows.

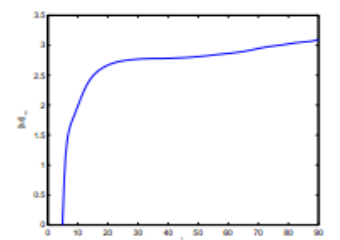

Fig. 4 Bifurcation of the equilibrium solution of Eq.4

So we deduce that there are also at least two equilibrium solutions for the Dirichlet problem Eq.4: $u=0, u=u_{\lambda}$, when $\lambda>\pi^{2} / 2$.

\section{References}

[1] D. R. Nelson and N. M. Shnerb, Non-Hermitian Localization and Population Biology.Phys. Rev. E, 58(1998), no. 2, 1383-1403.

[2] K. A. Dahmen, D. R. Nelson, and N. M. Shnerb, Life and death near a windy oasis.J. Math. Biol.,41(2000), no. 2, 1-23.

[3] R. A. Fisher, The wave of advance of advantageous genes. Ann. of Eugenics, 7(1937),355-369.

[4] J. D. Murray, Mathematical Biology. 2nd Edition,Springer, New York,(1993).

[5] V. M. Kenkre, Results from variants of the Fisher equation in the study of epidemics and bacteria. Proc.of.Amer.Phys.Soc., 342(2004), 242-248.

[6] M. A. Fuentes, M. N. Kuperman, V. M. Kenkre, Nonlocal Interaction Effects on Pattern Formation in Population Dynamics.Phys. Rev. Lett.91(2003), no. 15,158104/1-4.

[7] V. M. Kenkre, K. Lindenberg(Eds.), Patterns, Noise, and the Interplay of Nonlinearity and Complexity.AIP, New York,(2003).

[8] K. A.Dahmen, D. R. Nelson, N. M. Shnerb, Life and death near a windy oasis.J. Math. Biol., 41(2000), 1-23.

[9] V. M. Kenkre, M. N. Kuperman, Applicability of the Fisher equation to bacterial population dynamics.Phys. Rev. E67(2003) 051921.

[10] J. Shi, Lecture Notes on Reaction-Diffusion equations, chapter 4.

[11] L.N.Sun; J. Shi; Y.W.Wang, Existence and Uniqueness of Steady State Solutions of a Nonlocal Diffusive Logistic Equation. Zeitschrift fuer Angewandte Mathematik und Physik (ZAMP), 64 (2013), no. 4, 1267--1278. 